Revue d'histoire de l'Amérique française

REVUE D.HISTOIRE DE L'AMÉRIQUE FRANÇAISE

\title{
Une biographie politique et intellectuelle de Louis Francoeur
}

\section{Mathieu Noël}

Volume 66, numéro 3-4, hiver-printemps 2013

URI : https://id.erudit.org/iderudit/1025536ar

DOI : https://doi.org/10.7202/1025536ar

Aller au sommaire du numéro

\section{Éditeur(s)}

Institut d'histoire de l'Amérique française

\section{ISSN}

0035-2357 (imprimé)

1492-1383 (numérique)

Découvrir la revue

\section{Citer cet article}

Noël, M. (2013). Une biographie politique et intellectuelle de Louis Francoeur. Revue d'histoire de l'Amérique française, 66(3-4), 419-439.

https://doi.org/10.7202/1025536ar

\section{Résumé de l'article}

Cet article est une biographie politique et intellectuelle de Louis Francoeur (1895-1941), l'un des journalistes canadiens-français les plus influents des années 1930. Nous présentons sa carrière tout en portant une attention particulière à son parcours politique et intellectuel. D’abord intéressé par la vie ecclésiastique, il quitte l'ordre des Bénédictins au début des années 1920 pour entreprendre une carrière dans le journalisme et la politique. À partir de l'été de 1940, il anime à Radio-Canada l'émission quotidienne La situation ce soir, dans laquelle il présente et commente les actualités entourant la Deuxième Guerre mondiale. Louis Francoeur était un conservateur nationaliste et catholique, mais de tendance plus progressiste que traditionaliste. 


\title{
Une biographie politique et intellectuelle de Louis Francoeur
}

\author{
MATHIEU NoËL \\ Département d'histoire \\ Université du Québec à Montréal
}

RÉsumé - Cet article est une biographie politique et intellectuelle de Louis Francoeur (1895-1941), l'un des journalistes canadiens-français les plus influents des années 1930. Nous présentons sa carrière tout en portant une attention particulière à son parcours politique et intellectuel. D'abord intéressé par la vie ecclésiastique, il quitte l'ordre des Bénédictins au début des années 1920 pour entreprendre une carrière dans le journalisme et la politique. À partir de l'été de 1940, il anime à Radio-Canada l'émission quotidienne La situation ce soir, dans laquelle il présente et commente les actualités entourant la Deuxième Guerre mondiale. Louis Francoeur était un conservateur nationaliste et catholique, mais de tendance plus progressiste que traditionaliste.

ABSTRACT - This article is a political and intellectual biography about Louis Francoeur (1895-1941), one of the most influential French-Canadian journalists of the 1930s. His career is reviewed with a particular interest to his political and intellectual journey. First interested in consecrated life, he left the Benedictine Order in the early 1920s to pursue a career in journalism and politics. From the summer of 1940, he hosts at Radio-Canada the daily show La situation ce soir, during which he reports and comments the news surrounding World War II. We will illustrate that Louis Francoeur was a Catholic and a conservative nationalist with progressive trends, rather than traditionalist's.

$\mathbf{I}^{\mathrm{d}}$

dentifié à une histoire positiviste qui accordait trop d'importance à l'événement, le genre biographique a longtemps eu mauvaise presse. En 1974, Jacques Le Goff et Pierre Nora, dans le premier tome de Faire 
de l'histoire, présentaient la biographie comme un champ en dehors de l'histoire et comme le terrain "des vulgarisateurs de bas étage» et des "plumitifs de l'historiette»'. Dans l'historiographie française, les avis sur la biographie ont commencé à changer au début des années 1980, en raison du retour de l'événement et de la réhabilitation de l'histoire politique $^{2}$. Au Québec, toutefois, elle est encore peu utilisée par les historiens. Plusieurs politiciens et intellectuels marquants de notre histoire n'ont toujours pas été le sujet de biographies par des historiens professionnels. Pourtant, comme le souligne Jean-François Sirinelli, loin d'être réductrice, l'approche biographique contribue à une meilleure compréhension globale de l'histoire en permettant de lire en filigrane «les enjeux politiques d'une époque, les routes possibles qui s'ouvrent au choix individuel et les paramètres qui pèsent sur ce choix ${ }^{3} »$.

L'article qui suit est une biographie politique et intellectuelle de Louis Francoeur (1895-1941), l'un des journalistes canadiens-français les plus influents des années 1930. Il a écrit plusieurs centaines d'éditoriaux qui ont paru, principalement, dans Le Journal, L'Illustration, La Patrie du Dimanche et La Revue moderne ${ }^{4}$. De plus, il a été animateur de radio et analyste à Radio-Canada. À partir de l'été de 1940, Francoeur anime l'émission La situation ce soir, où il transmet les actualités militaires et tente d'expliquer les enjeux de la Deuxième Guerre mondiale aux Canadiens français. Selon Robert Rumilly, «tout le monde écoute son émission quotidienne ${ }^{5} »$. Francoeur décède le $1^{\text {er }}$ juin 1941 dans un accident d'automobile. Signe de sa popularité auprès des Canadiens français, environ 50000 personnes lui ont rendu un dernier hommage à l'Institut des sourdesmuettes à Montréal.

Malgré le fait qu'il ait été un acteur important dans l'histoire du journalisme au Québec, peu d'études existent sur Louis Francoeur. Au lendemain de son décès, quelques témoignages ont été publiés sous forme de

1. Jacques Le Goff et Pierre Nora, dir., Faire de l'histoire, tome 1, Nouveaux Problèmes (Paris, Gallimard, 1986), 15.

2. Guillaume Piketty a habilement analysé le "retour» du genre biographique en France. Voir Guillaume Piketty, «La biographie comme genre historique? Étude de cas», Vingtième siècle. Revue d'histoire, 63 (1999): 119-126.

3. Jean-François Sirenelli, dir., Dictionnaire de la vie politique française au XX $X^{e}$ siècle (Paris, PUF, 1995), vi. Cité dans G. Piketty, op. cit., 120.

4. André Versailles estime qu’entre 1922 et 1939, Francoeur aurait écrit plus de 6000 articles. André Versailles, «Louis Francoeur: journaliste de l'air», Radiomonde, 18 mai 1940, 5.

5. Robert Rumilly, Histoire de la province de Québec, tome 39 (Montréal, Fides, 1969), 75. 
brochures ${ }^{6}$. Également, l'on retrouve des repères biographiques dans des outils de références ${ }^{7}$ et des ouvrages plus généraux sur l'histoire de la radio $^{8}$. Néanmoins, mis à part ces quelques traces, rien n’a été écrit sur Francoeur. Cela s'explique par une raison fort simple: jusqu'à tout récemment, il n'y avait pas de sources primaires accessibles et aucune archive de Francoeur n'était disponible à la consultation. Jusqu'ici, seuls ses articles de journaux et quelques-unes de ses causeries radiophoniques pouvaient être consultés.

Or, la famille de Louis Francoeur a précieusement conservé ses archives. Il s'agit d'un fonds d'archives privé d'une très grande valeur. Les 8 boîtes comprennent des documents personnels, des agendas et journaux de bord, une correspondance d'environ 450 lettres $^{9}, 9$ discours électoraux, 4 albums renfermant la majorité de ses articles de journaux écrits dans les années 1920 et 1930, des causeries radiophoniques sur support audio, des documents administratifs de journaux qu'il a dirigés (Le Journal et L'Illustration) ainsi que ceux du Service d'urbanisme et de recherche de la Commission métropolitaine de Montréal, et des textes de différentes natures (des travaux scolaires, deux pièces de théâtre, des essais sur la spiritualité et sur l'histoire, etc.) Finalement, le fonds comprend plusieurs photographies d'époque.

Ces archives, jusqu' ici inconnues des historiens, constituent la principale source de cet article ${ }^{10}$. Notre objectif est de présenter la carrière de Louis Francoeur, tout en portant une attention particulière sur son parcours politique et intellectuel. Nous verrons que Francoeur était un conservateur nationaliste et catholique, mais de tendance plus progressiste que traditionaliste. Notre méthode s'inspire de celle de François Dosse; nous avons réalisé une lecture internaliste des écrits de Francoeur, tout en pri-

6. Claude-Henri Grignon, «Louis Francoeur, toujours vivant», Les Pamphlets de Valdombre, (avril-mai 1941) : 348-415; "Hommage à Louis Francoeur», Regards, (juillet-août 1941): 193-223; Blaise Orlier [pseudonyme de Guy Sylvestre], Louis Francoeur, journaliste (Ottawa, Les Éditions du Droit, 1941), 31 p.

7. Elzéar Lavoie, "La Situation ce soir, essais de Louis Francoeur», dans Maurice Lemire, dir., Dictionnaire des æuvres littéraires du Québec, tome III (Montréal, Fides, 1980), 914-917.

8. Pierre Pagé, Histoire de la radio au Québec: information, éducation, culture (Montréal, Fides, 2007), $488 \mathrm{p}$.

9. La majorité des lettres ont été écrites entre 1931 et 1941. Elles peuvent être divisées en trois catégories: famille, amis et correspondance avec le public.

10. Je tiens à remercier Madame Anne Francoeur de m'avoir autorisé à consulter et à utiliser sa collection d'archives privées de Louis Francoeur. 
vilégiant une démarche externaliste pour vérifier dans quels réseaux et dans quels milieux de sociabilité il évoluait ${ }^{11}$.

\section{UNE ENFANCE DANS LA TRADITION CATHOLIQUE}

Né à Montréal le 3 avril 1895, Louis Francoeur est le fils de Démétrius Francoeur (1866-1912), marchand de fourrures, et d'Ernestine Goulet (1869-1946). Après avoir quitté son mari quelque temps après la naissance de Louis, Mme Goulet et son fils ont trouvé refuge au couvent NotreDame-de-Lourdes, qui était à cette époque la maison-mère de la congrégation des Petites Filles de Saint-Joseph. Au même moment, elle est entrée au service de la maison de courtage L.-J. Forget et compagnie. Sa carrière professionnelle à la Bourse de Montréal nous est connue grâce à son témoignage rédigé en $1928^{12}$. Lorsqu'elle travaillait, Louis demeurait au couvent avec les religieuses. Bref, dès son plus jeune âge, il a évolué dans un milieu religieux. Sa mère était elle-même une fervente catholique, et elle espérait le voir entrer dans les ordres ${ }^{13}$.

Francoeur fait son cours classique au Collège Saint-Laurent, où il se lie d'amitié avec Claude-Henri Grignon. Grignon, alias Valdombre, se fera connaître en 1933 par la publication du roman à succès Un homme et son péché. Dans un article relatant leurs années au collège, Grignon décrit Francoeur comme un intellectuel qui avait toujours un livre à la main: «il dévorait tout depuis L'Île mystérieuse de Jules Verne jusqu'au Génie du christianisme de Chateaubriand ${ }^{14}$ ».

En 1913, après ses études classiques, Francoeur fait la rencontre du père Paul Vannier ${ }^{15}$. Celui-ci était en mission au Québec depuis quelques mois pour fonder une abbaye bénédictine de la congrégation de Solesmes. L'abbaye de Saint-Benoît-du-Lac, dans les Cantons-de-l'Est, voit le jour le 4 décembre 1912. Après quelques entretiens, Vannier convainc Francoeur d'adhérer à l'ordre bénédictin. Selon Mme Goulet, son fils a été attiré par la vie intellectuelle intense des Bénédictins et par la perspective d'un

11. François Dosse, La marche des idées. Histoire des intellectuels, histoire intellectuelle (Paris, La Découverte, 2003), 353 p.

12. Madame Francoeur [nom de plume d'Ernestine Goulet], Trente ans rue St-François-Xavier et ailleurs (Montréal, Éditions Édouard Garand, 1928), 134 p.

13. Dans les années 1910, Ernestine Goulet était une fidèle de l'abbaye de Saint-Benoît-du-Lac; son appartement, à Montréal, servait de gite pour les pères bénédictins en passage dans la métropole. Archives de Louis Francoeur, Texte inédit d’Ernestine Goulet intitulé Mes souvenirs de Saint-Benoît-du-Lac, 28 février 1945 .

14. C.-H. Grignon, op. cit., 354.

15. Les détails de leurs rencontres sont décrits par Ernestine Goulet. Archives de Louis Francoeur, Ernestine Goulet, Mes souvenirs de Saint-Benoît-du-Lac, 28 février 1945. 
voyage en Europe ${ }^{16}$. En septembre 1913, Francoeur part au prieuré de Conques, en Belgique, pour faire son noviciat ${ }^{17}$. Ce prieuré abritait alors la communauté des moines bénédictins de Saint-Wandrille, exilée de France à la suite de la loi du $1^{\text {er }}$ juillet $1901^{18}$.

\section{SES ANNÉES EN EUROPE, 1913-1923}

Les chroniques de l'abbaye de Saint-Wandrille nous informent que Francoeur a revêtu l'habit monastique le 11 novembre 1913, et qu'il a entrepris son noviciat canonique le 28 juin $1914^{19}$. Il semble s'être spécialisé en théologie et en histoire; ses archives comprennent des essais inédits sur l'histoire du christianisme, sur les controverses autour du jansénisme, sur l'histoire de l'abbaye de Saint-Wandrille et une synthèse sur la vie monastique. Dans celle-ci, Francoeur explique qu'après mûres réflexions, sa mission, en tant que moine, est d'entrer dans une union intime avec Dieu. Pour ce faire, il entend s'imposer «une vie de prière, d'obéissance et d'humiliation ${ }^{20} »$. Son noviciat est toutefois rapidement interrompu, ou à tout le moins perturbé, par la Grande Guerre.

Suivant le plan Schlieffen, les forces armées allemandes envahissent la Belgique en août 1914, pour permettre une éventuelle invasion de la France via les Ardennes. Au début du conflit, le prieuré de Conques est transformé en hôpital militaire pour soigner les blessés belges et français. Par contre, dès la fin du mois d'août, les Allemands réussissent à prendre le bourg d'Herbeumont, et par le fait même, le prieuré de Conques. Les moines et les novices bénédictins de Saint-Wandrille sont considérés comme des prisonniers civils et seront confinés dans leur domaine prieural pendant toute la durée de la guerre. Après la prise d'Herbeumont, le major commandant Turner, du $50^{\mathrm{e}}$ Landsturm hessois, réquisitionne le prieuré pour y établir ses quartiers et pour y loger une cinquantaine d'hommes.

16. Ibid.

17. Archives de Louis Francoeur, Lettre de Louis Francoeur au père Paul Vannier, 23 juillet 1913.

18. La loi française du $1^{\text {er }}$ juillet 1901 restreignait la liberté d'association des congrégations religieuses. Conséquemment, plusieurs d'entre elles se sont réfugiées dans d'autres pays. La communauté des moines bénédictins de Saint-Wandrille est demeurée au prieuré de Conques pendant trente ans, soit de 1901 à 1931.

19. Les mentions à Louis Francoeur dans les chroniques de l'abbaye de Saint-Wandrille ont été photocopiées et envoyées à Jacques Francoeur, le fils de Louis, en 1993. Archives de Louis Francoeur, Lettre du père Hugues Leroy à Jacques Francoeur, 18 novembre 1993.

20. Archives de Louis Francoeur, Manuscrit inédit du frère Louis Francoeur intitulé Essai de synthèse sur la vie monastique. 
Dans un témoignage rédigé en $1936^{21}$, Francoeur explique que l'occupation de Conques «bouleversa de fond en comble la vie silencieuse du domaine et de ses habitants, [mais quelle] leur apporta la sécurité2 ${ }^{22}$. Les Bénédictins n'avaient plus à craindre "les violences et les caprices des troupes de passage, puisque rien ne pouvait se faire sur les lieux sans la permission du commandant résidant ${ }^{23}{ }^{2}$. Étant le seul à parler l'anglais, langue que parle le commandant allemand, Francoeur est nommé interprète de l'officier supérieur. Si l'on se fie à son récit, une certaine complicité naît entre lui et le major Turner. C’est possiblement lors de cette période que Francoeur apprend l'allemand. À l'automne de 1914, le 50 Landsturm est remplacé par un contingent de Saxons, et à la fin de l'hiver de 1915, les Saxons sont à leur tour appelés au front - aucune autre troupe n’occupera le prieuré avant $1918^{24}$. Bien qu'ils soient toujours confinés, les Bénédictins peuvent alors reprendre la formation des novices. C’est donc en période de guerre, plus précisément le 10 février 1917, que Francoeur fit sa profession simple perpétuelle ${ }^{25}$.

Parallèlement à la poursuite de son noviciat, Francoeur nous informe, toujours dans son témoignage de 1936, qu'il a fait de l'espionnage de guerre pour les services secrets britanniques ${ }^{26}$. Au printemps de 1915, son aide est sollicitée par un jeune espion qui prenait des photos pour le futur rapport Bryce ${ }^{27}$. La mission de Francoeur était de retenir le plus d'informations possible sur les actions allemandes dans la région, pour ensuite livrer les renseignements récoltés à l'espion-photographe lors de ses passages à Conques. Ce travail a duré quelques mois. Selon Jacques Francoeur, son père aurait été démasqué et condamné à mort par les Allemands ${ }^{28}$. Toutefois, rien ne se produisit et Francoeur demeure reclus au prieuré

21. Louis Francoeur, "Souvenirs de l'invasion allemande en Ardenne belge 1914 », La Patrie du Dimanche, série d'articles publiés du 15 mars 1936 au 14 juin 1936.

22. Louis Francoeur, "Souvenirs de l'invasion allemande en Ardenne belge 1914 », La Patrie du Dimanche, 26 avril 1936, 18.

23. Ibid.

24. Louis Francoeur, "Souvenirs de l'invasion allemande en Ardenne belge 1914 », La Patrie du Dimanche, 3 mai 1936, 19.

25. Archives de Louis Francoeur, Lettre du père Hugues Leroy à Jacques Francoeur, 18 novembre 1993.

26. Louis Francoeur, "Souvenirs de l'invasion allemande en Ardenne belge 1914 », La Patrie du Dimanche, 10 mai 1936, 19.

27. James Bryce avait été désigné par le gouvernement anglais pour enquêter sur de supposées atrocités allemandes commises en Belgique. Pour mener son enquête, Bryce avait eu recours à l'espionnage.

28. Archives de Louis Francoeur, Texte de l'allocution de Jacques Francoeur à la soirée en hommage à Louis Francoeur organisée par le Conseil de Presse, 27 novembre 1989. 
jusqu'en novembre 1918. Après la guerre, il est envoyé en France où il est soigné pour la grippe espagnole.

En 1919, Francoeur revient quelques mois au Québec pour visiter sa mère. Durant ce séjour, il tient un journal que nous avons retrouvé dans ses archives personnelles ${ }^{29}$. Nous constatons qu'il participe alors à la vie religieuse de l'abbaye de Saint-Benoît-du-Lac: il sert la messe, il participe aux chants grégoriens et il visite des curés de différentes paroisses. Il en profite également pour renouer avec son ami Claude-Henri Grignon. Ce dernier explique que Francoeur, vêtu de son scapulaire noir, avait tenté de le convaincre d'abandonner les idées romantiques pour revenir dans le «droit chemin» du catholicisme ${ }^{30}$. Francoeur aurait alors été sous le charme des idées de Louis Veuillot, journaliste ultramontain français du $\mathrm{XIX}^{\mathrm{e}}$ siècle ${ }^{31}$. Bref, lors de son voyage au Québec - du 11 février à la fin de juin 1919 - rien ne laissait présager sa décision de quitter l’ordre bénédictin à son retour en Belgique.

En effet, le 10 juillet 1919, Francoeur annonce à ses supérieurs qu’il quitte la vie ecclésiastique ${ }^{32}$. Selon Robert Prévost, Francoeur abandonne son projet de vie religieuse à la suite d'un différend avec ses professeurs à propos d'une thèse sur le péché qu'ils ont jugée non orthodoxe ${ }^{33}$. Pour sa part, le père Émile Legault affirme que Francoeur a quitté les Bénédictins en "pleine crise existentielle», puisqu’il n’appréciait pas certains aspects trop conformistes de l'Église ${ }^{34}$.

Après son départ de l'ordre de Saint-Benoît, Francoeur vit environ trois ans en France - principalement à Paris. Il s'y initie aux lettres et à l'édition, tout en demeurant dans les milieux catholiques. À Paris, il est accueilli par le prêtre Jean Verdier, qui sera archevêque de la région parisienne de 1929 à 1940. Ce dernier l'encourage à poursuivre son bénévolat au sein de l'Église catholique, ce que fera Francoeur en siégeant sur deux comités d'étude: l'un sur l'art religieux et la liturgie, l'autre sur le mou-

29. Archives de Louis Francoeur, Agenda de Louis Francoeur, 1919.

30. C.-H. Grignon, op. cit., 373.

31. Ibid., 369.

32. Il s'agit de la date inscrite dans les chroniques de l'abbaye de Saint-Wandrille: Archives de Louis Francoeur, Lettre du père Hugues Leroy à Jacques Francoeur, 18 novembre 1993. Par contre, il s'agit peut-être d'une erreur, puisque dans son journal de 1919, qui se termine le 30 juillet, Francoeur ne fait pas mention de ce choix et il semble poursuivre ses activités monastiques. Selon Claude-Henri Grignon, c'est plutôt au début de l'année 1920 que Francoeur quitte l’ordre: C.-H. Grignon, op. cit., 373.

33. Robert Prévost, Mon tour de jardin (Québec, Septentrion, 2002), 41.

34. Émile Legault, «Francoeur, chrétien», Regards (juillet-août 1941): 210-211. 
vement des idées ${ }^{35}$. Verdier lui trouve différents emplois; il sera même quelque temps le précepteur des enfants de l'impératrice Zita, alors en exil de l'empire austro-hongrois ${ }^{36}$.

À l'hiver de 1920, Francoeur écrit à Claude-Henri Grignon pour l'informer qu'il travaille comme publicitaire pour la maison d'édition Gabriel Beauchesne et qu'il est secrétaire de rédaction de Lumen, une revue d'enseignement supérieur ${ }^{37}$. De plus, il milite dans l'Association catholique de la jeunesse française $(\mathrm{ACJF})^{38}$. Toujours selon Grignon, Francoeur profite de ses années à Paris pour fréquenter différents cercles littéraires et, à partir de novembre 1920, il est administrateur de la revue Les Lettres ${ }^{39}$. Cette revue d'idées, dirigée par Gaëtan Bernoville (1889-1960), réunissait de jeunes intellectuels rattachés au mouvement de la "renaissance littéraire catholique $~^{40}$. Ceux-ci demandaient à ce que les hommes de lettres puissent prendre part à la défense et à l'ascension de l'Église catholique. C'est dans cette revue que Francoeur publie ses premiers articles ${ }^{41}$. Bref, lors de ses années en France, Francoeur s'intéresse à l'écriture et il reste un catholique pratiquant.

\section{LE RETOUR D'EUROPE ET LES DÉBUTS DANS LE JOURNALISME, I923-1929}

En 1923, Louis Francoeur revient au Québec. Sa mère aurait alors usé de ses contacts du temps à la Bourse, où elle travaillait, pour lui dénicher un emploi de journaliste à La Patrie ${ }^{42}$. Pendant quatre ans, il est courriériste parlementaire pour ce journal, fonction qui lui permet de se familiariser avec les rouages de la politique. Il passe ensuite au Montreal Star, toujours comme courriériste parlementaire jusqu'en 1929.

À La Patrie et au Montreal Star, Francoeur rédige aussi des chroniques littéraires. Il faut dire que durant cette période, le jeune journaliste continue de s'intéresser à la littérature. Le 13 novembre 1924, il se joint à l'École lit-

35. Archives de Louis Francoeur, Texte de l'allocution de Jacques Francoeur à la soirée en hommage à Louis Francoeur organisée par le Conseil de Presse, 27 novembre 1989.

36. Ibid.

37. Lettre de Louis Francoeur à Claude-Henri Grignon, 5 mars 1920, citée dans C.-H. Grignon, op. cit., 373-374.

38. Ibid.

39. C.-H. Grignon, op. cit., 375.

40. Concernant la revue Les Lettres et le mouvement de la "renaissance littéraire catholique», voir Hervé Serry, «Déclin social et revendication identitaire: la "renaissance littéraire catholique” de la première moitié du XX" siècle», Sociétés contemporaines, 44, (2001): 91-109.

41. Les archives de Louis Francoeur comprennent quelques documents relatifs aux Lettres et à la Semaine des écrivains catholiques, un congrès annuel organisé par les collaborateurs de la revue.

42. C’est du moins ce qu'affirme André Versailles dans son article: «Louis Francoeur: journaliste de l'air», Radiomonde, 18 mai 1940, 5. 
téraire de Montréal. À cette époque, celle-ci est toujours marquée par des querelles intestines entre les exotiques, partisans de l'art universel, et les régionalistes, partisans du traditionalisme et d'une littérature nationale ${ }^{43}$. Nous aurions pu penser qu'un catholique pratiquant, qui venait tout juste de quitter le mouvement de la "renaissance littéraire catholique», aurait pris parti pour les régionalistes. Or, c'est plutôt du côté des exotiques que l'on retrouve Francoeur. Sa présence est toutefois de courte durée, puisque le 26 février 1925, Victor Barbeau, Roméo Bouchard, Berthelot Brunet, Philippe Panneton (Ringuet) et lui quittent l'École littéraire de Montréal à la suite d'une ultime dispute avec les régionalistes ${ }^{44}$.

En 1924, Francoeur publie un premier livre, Littératures... à la manière $d e^{45}$, écrit en collaboration avec Philippe Panneton (Ringuet). Il s'agit d'un livre de pastiches, où les auteurs se moquent amicalement de la façon d'écrire de certaines personnalités. Ce livre semble avoir connu un succès critique et commercial; les auteurs ont même reçu le prix David de 1924 et le livre a été réédité à quatre reprises, en 1925, 1941, 1942 et $1943^{46}$.

Louis Francoeur se marie à Adèle Gervais le 16 juin 1924. Le couple aura un seul enfant, Jacques (1925-2005), qui fera carrière comme journaliste et éditeur de journaux.

\section{DIRECTEUR DU JOURNAL, I929- 1934}

En décembre 1929, Louis Francoeur quitte son emploi au Montreal Star et il déménage à Québec, où il participe à la fondation de l'hebdomadaire Le Journal. Financé par des proches du Parti conservateur fédéral, ce journal voulait défendre les positions de Richard B. Bennett et de Camillien Houde, alors les chefs conservateurs à Ottawa et à Québec. Francoeur y est nommé rédacteur en chef. Dans les faits, il est l'auteur de pratiquement tout le contenu du journal: il rédige l'éditorial, il rapporte les actualités politiques et il signe les chroniques littéraires. Nous soupçonnons même qu’il rédigeait parfois anonymement les chroniques sportives et féminines, car plusieurs d'entre elles font partie de ses albums de spicilèges.

43. Pour une analyse détaillée du conflit entre régionalistes et exotiques, voir Annette Hayward, $L a$ querelle du régionalisme au Québec (1904-1931). Vers l'autonomisation de la littérature québécoise (Ottawa, Le Nordir, 2006), $622 \mathrm{p}$.

44. François Couture et Pierre Rajotte, «L’École littéraire de Montréal et ses mythes », Études françaises, 36, 3, (2000): 182 .

45. Louis Francoeur et Philippe Panneton, Littératures... à la manière de (Montréal, Éditions Édouard Garand, 1924), 132 p.

46. Littératures... à la manière de semble avoir été redécouvert lors du décès de Francoeur (en 1941), comme en témoignent les rééditions de 1941, 1942 et 1943. 
Lors des premiers mois de publication, Francoeur participe activement à la précampagne électorale fédérale en critiquant vigoureusement le gouvernement libéral. Dans ses articles, il fait porter la responsabilité de la crise économique sur les libéraux. Selon lui, Mackenzie King a aggravé le problème du chômage au Canada en laissant entrer des immigrants communistes $^{47}$. Par ailleurs, l'absence de mesures protectionnistes pour venir en aide aux industriels et aux agriculteurs canadiens nuirait à la reprise économique. Francoeur estime que pour sortir de la crise, les Canadiens doivent élire un gouvernement conservateur à Ottawa ${ }^{48}$. Or, une fois les conservateurs au pouvoir, il expliquera que les mauvaises décisions du gouvernement précédent représentent un fardeau aux bonnes volontés de Bennett ${ }^{49}$.

Le Journal accorde également une grande considération à la politique provinciale. Chaque semaine, le gouvernement libéral d'Alexandre Taschereau est attaqué. Comme le souligne l'historien Richard Jones, deux accusations sont portées contre les libéraux : la corruption et l'exploitation pernicieuse des ressources naturelles ${ }^{50}$. Également, Francoeur considère que par ses inactions, Taschereau participe à l'expansion du bolchévisme au Québec ${ }^{51}$. Lors de la victoire électorale libérale de 1931, Le Journal tentera, sans succès, de prouver que les libéraux ont remporté les élections de manière frauduleuse. Pendant plusieurs semaines, Francoeur mènera une campagne contre le projet de loi Dillon, qui visait à restreindre les contestations d'élections.

Bien que la mission du Journal était avant tout de défendre les conservateurs et de s'en prendre aux libéraux, Francoeur abordait parfois d'autres sujets, ce qui nous permet de mieux saisir sa pensée. La «jeunesse" est l'un des thèmes auquel il s'intéresse. Dans une série d'articles publiée en 1933, Francoeur déplore que la jeunesse soit exclue de «l'organisation sociale» et des solutions de sortie de crise ${ }^{52}$. Négligée et désabusée, elle se tournerait vers des idéologies extrêmes, telles que le fascisme et le communisme. Dans l'un de ses textes, il rappelle que Mussolini

47. Louis Francoeur, «La politique», Le Journal, $1^{\mathrm{er}}$ mars 1930.

48. Louis Francoeur, «M. Bennett», Le Journal, 24 mai 1930.

49. Louis Francoeur, "La politique», Le Journal, 30 août 1930.

50. Richard Jones, "Le Journal, 1929-1932», dans Fernand Dumont, Jean-Paul Montminy et Jean Hamelin, dir., Idéologies au Canada français, 1930-1939 (Québec, Les Presses de l’Université Laval, 1978), 170 .

51. Louis Francoeur, «Bolchévisme», Le Journal, 31 janvier 1931.

52. Louis Francoeur, «La crise de la jeunesse», Le Journal, série d’articles publiés du 19 mai 1933 au 27 juillet 1933. 
a bâti sa puissance grâce à la foi qu'il inculquait aux jeunes. Bref, le fait d'ignorer la jeunesse risquerait d'engendrer une "grave menace sociale ${ }^{53}$ ". Au terme de sa série d'articles, il propose aux conservateurs une stratégie qui permettrait de rallier la jeunesse canadienne-française. Selon lui, comme les jeunes se sentent laissés pour compte, le Parti conservateur aurait avantage à adopter un programme d'action nationale qui leur accorderait une place de choix. D'ailleurs, au même moment, Francoeur essaie de convaincre le groupe nationaliste des Jeune-Canada d'adhérer au parti ${ }^{54}$.

Par ailleurs, Francoeur s'intéresse au système d'enseignement dans la province de Québec. En 1933, il affirme que les faiblesses du système d'éducation font en sorte que le peuple n'a pas de curiosité intellectuelle et que les classes dirigeantes sont "d'une apathie indécrottable ${ }^{55}$ ». Il souhaite que l'Église et l'État organisent des États généraux sur l'éducation pour examiner les problèmes et proposer des réformes. Dans les années suivantes, Francoeur reviendra souvent sur cette réforme souhaitable du système d'enseignement. Par exemple, dans une allocution à la Société Saint-Jean-Baptiste de Québec, il proposera de revoir le modèle des collèges classiques pour offrir un enseignement pratique et moderne qui miserait sur la spécialisation des étudiants selon leurs talents ${ }^{56}$. Ainsi, bien qu'il soit à la tête d'un journal conservateur et que sa tâche soit avant tout de malmener les libéraux, Francoeur a la liberté d'exprimer et de proposer des idées relativement progressistes.

\section{MILITANTISME CONSERVATEUR}

En plus de diriger Le Journal, Francoeur milite dans le camp conservateur. En 1930, il accompagne le premier ministre Bennett à la conférence impériale de Londres où il agit en tant que secrétaire de la délégation canadienne $^{57}$. De 1930 à 1933, il est membre de l'exécutif régional de Québec du Parti conservateur provincial. Puis, lors des élections provinciales de 1931, il est candidat conservateur dans la circonscription de Montmorency contre nul autre que le premier ministre sortant, Louis-Alexandre Taschereau. Son objectif est d'obliger Taschereau à faire campagne dans

53. Louis Francoeur, «La crise de la jeunesse », Le Journal, 26 mai 1933.

54. Le Journal, 10 mars 1933; Le Journal, 21 avril 1933.

55. Louis Francoeur, «Enfin!», Le Journal, 21 décembre 1933.

56. Archives de Louis Francoeur, Texte d'une allocution prononcée à la Société Saint-Jean-Baptiste de Québec, 5 novembre 1939.

57. Ses archives privées comprennent de nombreux documents relatifs à la conférence impériale de Londres. 
son comté. Dans cet affrontement électoral, Francoeur reprend sensiblement les mêmes attaques qu'il menait dans Le Journal. Plus précisément, il reproche à Taschereau «le sacrifice des richesses naturelles, l'empiètement des trusts sur les privilèges du peuple, le sacrifice du progrès de la province à de gros intérêts et un favoritisme ébranlé dans son administration $^{58}$ ». De plus, il critique les promesses libérales de construire des routes et des ponts avec des fonds qui, selon lui, pourraient être utilisés pour doter la province d'un plan de développement agricole et pour aider les industries affectées par la crise économique ${ }^{59}$. Finalement, Taschereau sera réélu dans Montmorency en obtenant 1949 votes, contre 1368 pour Francoeur. À l'échelle provinciale, les libéraux remportent 79 sièges, tandis que les conservateurs n'en récoltent que 11.

Battu dans les deux circonscriptions où il se présentait ${ }^{60}$, Camillien Houde quitte la direction du Parti conservateur. Louis Francoeur est alors pressenti pour être candidat. Il est d'abord sollicité par l'influent groupe de Québec mené par Noël Dorion ${ }^{61}$ et, en septembre 1932, il reçoit l'appui du club conservateur ouvrier de Québec-Est ${ }^{62}$. Lors d'une assemblée partisane, Francoeur affirme que le Parti conservateur doit se réorienter vers un nationalisme plus revendicateur ${ }^{63}$. Par contre, pendant la même assemblée, il semble hésiter à se porter candidat. Selon lui, il n'aurait pas les qualités requises en finance et en droit pour être un bon premier ministre.

Finalement, il se ralliera à l'équipe de Maurice Duplessis ${ }^{64}$. Cet appui n'est pas anodin puisqu'il est un journaliste connu et respecté dans les rangs conservateurs, et qu'il est membre du comité exécutif du congrès de Sherbrooke où se déroulera l'élection du nouveau chef ${ }^{65}$. En octobre 1933, Duplessis est élu à la tête du Parti conservateur du Québec.

58. «M. Dupré avec M. Foley à Donnacona ", L'Évènement, 15 août 1931.

59. Ibid.

60. À cette époque, un candidat pouvait se présenter à la fois dans plusieurs circonscriptions à un même scrutin. La Loi électorale interdit les candidatures multiples depuis 1952.

61. Selon Conrad Black, le groupe de Noël Dorion aurait d'abord sollicité la candidature de Louis Francoeur, mais à la suite du refus de ce dernier, le groupe aurait porté son dévolu sur Maurice Duplessis. Voir Conrad Black, Maurice Duplessis (Montréal, Les Éditions de l’Homme, 1999), 91.

62. "Un vote de confiance à M. Francoeur", L'Action catholique, 30 septembre 1932.

63. Ibid.

64. Louis Francoeur aurait accompagné Pierre Bertrand, Jean Mercier et Noël Dorion à Trois-Rivières pour convaincre Duplessis de se présenter. Voir Louis Francoeur, "Duplessis intime», La Patrie du Dimanche, 6 septembre 1936, 6.

65. D’ailleurs, les adversaires de Duplessis accusèrent ce dernier d'avoir noyauté le comité organisateur du congrès. Voir Conrad Black, op. cit., 93. 


\section{RÉDACTEUR EN CHEF DE L'ILLUSTRATION, I 934 - I 935}

En février 1934, Louis Francoeur quitte Le Journal de Québec et fait ses débuts comme rédacteur en chef à L'Illustration, un quotidien bleu de Montréal. Fondé en 1930 par Eugène Berthiaume et les frères Lucien et Fernand Dansereau, ce journal appuyait ouvertement les politiques conservatrices $^{66}$. L'Illustration s'attelait à défendre la fin de mandat des conservateurs fédéraux, soutenait la candidature de Houde à la mairie de Montréal et supportait les conservateurs provinciaux de Duplessis. Le passage du Journal à L'Illustration représente donc une continuité idéologique et politique. D’ailleurs, Francoeur reprend dans la page éditoriale du tabloïd montréalais la chronique politique qu’il rédigeait dans son ancien journal, une chronique qu'il intitule «Maîtres chez nous». Il y livre des textes résolument nationalistes. Régulièrement, il appelle les Canadiens français à reprendre le contrôle de la province de Québec. Le 3 août 1934, il écrit:

Le Canadien français doit être maître en sa province, il doit prendre tous les moyens de reconquérir ce qu'il a perdu, il doit restituer à sa petite patrie son âme française et son apparence française qu'une majorité de 93 pour cent abdique virtuellement si elle reconnaît que toute sa vie politique, économique et sociale doit continuer d'être dominée par la minorité de sept pour cent ${ }^{67}$.

Quelques jours plus tard, il signe un autre texte sur l'importance pour les Canadiens français de s'affirmer et de prendre leur place:

[Il faut montrer] au reste du pays et aux Anglo-canadiens de la province que nous entendons devenir les maîtres chez nous, nous faire respecter et faire prévaloir notre intérêt collectif sur l'intérêt des autres. [...] Depuis quand des gens de sang français se laissent-ils, chez eux, exploiter et humilier sans rien dire, par une minorité qui sait les distraire et les diviser sur des questions de politiquette $^{68}$ ?

Sans aller jusqu'à appuyer ouvertement l'idée de l'indépendance du Québec, Francoeur encourage des groupes de jeunes qui proposent cette idée (les Jeune-Canada et Vivre) ${ }^{69}$, et il décrit la Confédération comme une «grande déception» et un «marché dans lequel [les Canadiens français]

66. Mentionnons qu'au moment où il était chef du Parti conservateur provincial, Camillien Houde a détenu quelque temps des parts dans cette entreprise de presse. Toutefois, lors de l'embauche de Francoeur, Houde ne fait plus partie des actionnaires de L'Illustration.

67. Louis Francoeur, «La Politique. Maîtres chez nous», L'Illustration, 3 août 1934, 4.

68. Louis Francoeur, «La Politique. Maîtres chez nous», L'Illustration, 7 août 1934, 4.

69. Louis Francoeur, «Vivre», L'Illustration, 11 décembre 1934, 4. 
ont été les dupes ${ }^{70}$. De plus, il se demande pourquoi une éventuelle déclaration d'autonomie est un sujet tabou au Québec ${ }^{71}$.

Toujours dans la chronique "Maîtres chez nous», Francoeur fait le procès du Parti libéral du Québec. En 1934, il semble prendre un vilain plaisir à présenter les divisions et les disputes internes au Parti libéral ${ }^{72}$. Selon lui, plusieurs libéraux ne croient plus en l'honnêteté de Taschereau, et c'est ce désaveu qui aurait conduit au schisme libéral et à la fondation de l'Action libérale nationale (ALN) ${ }^{73}$. Ce nouveau parti, dirigé par Paul Gouin, subit lui aussi initialement les foudres du rédacteur en chef de L'Illustration. Francoeur estime que l'ALN a copié le programme du Parti conservateur en y ajoutant quelques idées radicales qui sont irréalisables ${ }^{74}$. Sans surprise, lorsque les conservateurs de Duplessis amorceront un rapprochement avec les actionnistes de Gouin, Francoeur cessera de malmener l'ALN.

À la barre de L'Illustration, Francoeur s'intéresse de près au Parti conservateur fédéral. En septembre 1934, il publie une série d'articles où il propose ses idées pour réorganiser le parti. Tout d’abord, comme il le recommandait l'année précédente dans Le Journal, Francoeur croit que le parti doit accorder une plus grande importance aux jeunes et se munir d'une véritable aile jeunesse inspirée du modèle de la Jeunesse libérale ${ }^{75}$. Ensuite, il suggère que le parti se dote d'un bureau de presse et de propagande apte à contrecarrer la "presse rouge». Selon lui, la sympathie du Journal et de L'Illustration à la cause conservatrice est loin de suffire pour faire contrepoids à la machine libérale ${ }^{76}$. Finalement, Francoeur prône un renouvellement des idées et des arguments; il estime que les conservateurs doivent cesser de baser leurs arguments sur les réalisations passées de leurs anciens chefs John A. Macdonald et George-Étienne Cartier ${ }^{77}$. Les propositions de Francoeur visent à moderniser le Parti conservateur qu'il juge, sur certains aspects, dépassé.

70. Louis Francoeur, «La Politique. Maîtres chez nous », L'Illustration, 10 août 1934, 4.

71. Ibid.

72. Louis Francoeur, "La Politique. Maîtres chez nous», L'Illustration, 25 septembre 1934, 4.

73. Louis Francoeur, «La Politique. Maîtres chez nous», L'fllustration, 28 juillet 1934, 4.

74. Louis Francoeur, «La Politique. Maîtres chez nous», L'Illustration, 3 août 1934, 4 ; Louis Francoeur, «La Politique. Maîtres chez nous », L'Illustration, 6 août 1934, 4.

75. Louis Francoeur, «La crise du Parti conservateur», L'Illustration, 27 septembre 1934, 4.

76. Louis Francoeur, «La crise du Parti conservateur», L'Illustration, 28 septembre 1934, 4.

77. Louis Francoeur, «La crise du Parti conservateur», L'Illustration, 29 septembre 1934, 4. 


\section{CANDIDAT POUR LE PARTI DE LA RECONSTRUCTION DU CANADA}

Quelques mois après avoir présenté ses idées pour réformer le Parti conservateur fédéral, Francoeur rompt ses liens avec ce parti en raison d'un différend avec le premier ministre Richard B. Bennett. À ses amis, il expliquera être «sorti ouvertement du parti en 1935, à la suite d'une lettre stupide où le gros Bennett [lui] disait que les griefs des Canadiens français "did not deserve a reply"78 ". Déçu de voir les Canadiens français négligés par les conservateurs fédéraux, Francoeur quitte le parti pour se rallier au Parti de la reconstruction du Canada (PRC) ${ }^{79}$.

Ce parti fédéral a été fondé en 1935 par Henry Herbert Stevens (18781973) et d'autres conservateurs en dissension parce que le gouvernement ne voulait pas appliquer les recommandations de la Commission royale d'enquête sur les écarts de prix $^{80}$. Les membres du PRC proposent la création d'un programme national de logement, l'adoption d'un système de travaux publics pour donner des emplois aux chômeurs et ils s'engagent à s'opposer à la participation du Canada dans une éventuelle guerre européenne. Francoeur partageait sans doute plusieurs de ces idées, mais dans une causerie radiophonique, il explique avoir adhéré au PRC parce qu'il avait reçu la garantie de Stevens que le parti défendrait réellement les intérêts des Canadiens français à Ottawa ${ }^{81}$.

Aux élections générales de 1935, Louis Francoeur est candidat du PRC dans la circonscription montréalaise de Saint-Jacques ${ }^{82}$, occupée depuis 1920 par le député libéral - et ancien maire de Montréal - Fernand Rinfret. Deux enjeux ressortent de cette campagne: la défense des chômeurs de Saint-Jacques et la défense des Canadiens français à Ottawa. Concernant la question du chômage, Francoeur soutient l'idée de son chef d'adopter un système de travaux publics pancanadien pour créer des emplois. De plus, il propose de limiter l'immigration, puisque selon lui, les nouveaux arrivants risquent de concurrencer les chômeurs de Saint-Jacques ${ }^{83}$. En ce qui concerne la défense des Canadiens français, Francoeur affirme que le PRC est le seul parti qui protégera véritablement les droits des franco-

78. Archives de Louis Francoeur, Lettre de Louis Francoeur à Maurice Dupré, 12 avril 1938.

79. Mentionnons qu'en changeant d'allégeance politique, Francoeur quitte son emploi à L'Illustration.

80. Il s'agissait de différentes mesures visant à limiter le pouvoir d'achat des grands magasins et des magasins à succursales pour qu'ils cessent d'obliger les fabricants à vendre à petits prix.

81. Archives de Louis Francoeur, Texte d'une causerie prononcée par Louis Francoeur à la radio, 12 octobre 1935.

82. Mentionnons que le beau-père de Francoeur, Honoré Gervais (1864-1915), a été député de SaintJacques de 1904 à 1911 .

83. Archives de Louis Francoeur, Texte d'un discours électoral de Louis Francoeur, 1935. 
phones dans la Confédération. Selon lui, son parti vient «avec un message nouveau, un message de libération populaire, un message d'affirmation française ${ }^{84}$ ». Dans l'un de ses discours, il prend l'engagement, s'il est «élu, [de] jouer à Ottawa le rôle de chien de garde pour tout ce qui est d'intérêt canadien-français ${ }^{85}$ ». Il y explique vouloir reprendre le flambeau d'Armand Lavergne $^{86}$, décédé quelques mois plus tôt. Francoeur mise donc sur une approche populiste et nationaliste pour se faire élire. Il termine néanmoins deuxième en récoltant $27,77 \%$ des voix, derrière Fernand Rinfret, qui lui en obtient $60,46 \%$.

\section{IDÉOLOGUE DE L’UNION NATIONALE}

Malgré sa rupture avec les conservateurs fédéraux, Francoeur demeure dans le giron des conservateurs provinciaux. Entre 1934 et 1936, il collabore régulièrement avec le Parti conservateur du Québec puis avec l'Union nationale. Tout d'abord, il participe à l'écriture du Catéchisme des électeurs ${ }^{87}$, le premier programme officieux de l'Union nationale. Sous forme de questions réponses, cet instrument de propagande expliquait le plus simplement possible pourquoi les électeurs devaient défaire le gouvernement libéral et voter pour l'Union nationale. Par exemple, à la question numéro 50 sur «Comment renverser le régime Taschereau?», le Catéchisme répond: «En votant à cette élection provinciale pour les candidats de l'Union nationale qui groupe tous les libéraux libres, les conservateurs libres et tous les indépendants et tous les véritables patriotes de la Province en un parti populaire semblable à celui qui porta autrefois Mercier au pouvoir ${ }^{88}$.» Ce Catéchisme a été largement distribué dans toute la province.

Par ailleurs, dans le cadre des élections de 1935 et de 1936, Francoeur rédige des discours pour des candidats et il prononce des causeries partisanes à la radio. Par exemple, le 11 août 1936, il s'adresse aux anglophones de Montréal par le biais de la station CFCF pour leur demander de faire

84. Archives de Louis Francoeur, Texte d'une causerie prononcée par Louis Francoeur à la radio, 12 octobre 1935 .

85. Archives de Louis Francoeur, Texte d'un discours électoral de Louis Francoeur, 1935.

86. Armand Lavergne est un nationaliste qui s'est fait connaître au début du XX ${ }^{\mathrm{e}}$ siècle par ses luttes pour la défense de la langue française, notamment alors qu'il siégeait à la Chambre des communes de 1904 à 1908.

87. Louis Dupire, Louis Francoeur, Roger Maillet et Édouard Masson, Le Catéchisme des électeurs (Montréal, J.-B. Thivierge \& fils éditeurs, 1935), 128 p.

88. Exemple tiré d'Alain Lavigne, Duplessis, pièce manquante d'une légende. L'Invention du marketing politique (Québec, Septentrion, 2012), 35. 
confiance à Duplessis et de chasser les libéraux ${ }^{89}$, tandis que deux jours plus tard, il présente certaines idées et les valeurs de l'Union nationale sur les ondes de CHLP $^{90}$. Lors des élections de 1936, Francoeur met aussi sa plume au service de l'Union nationale en signant quelques textes partisans dans La Patrie du Dimanche $e^{91}$.

Louis Francoeur a donc joué un rôle important dans les débuts de l’Union nationale. Par contre, il sortira déçu et amer des élections de 1936. En effet, il souhaitait être candidat dans la circonscription de MontréalMercier. Sa correspondance montre qu'après les élections de 1935, il tentait d'obtenir des appuis en vue d'une éventuelle investiture dans le comté $^{92}$. Toutefois, le parti a préféré appuyer la candidature de Gérard Thibeault. Quelques années plus tard, Francoeur expliquera s'être détourné de la politique active après les élections de 1936, puisqu'il n'appréciait pas les méthodes de "gestapo» utilisées par la garde rapprochée de Duplessis ${ }^{93}$.

À l'automne de 1936, Louis Francoeur fait une croix sur une éventuelle carrière politique. Il se consacre tout d'abord à la réalisation du numéro dominical de La Patrie - il est directeur de La Patrie du Dimanche depuis le printemps. Puis, lorsque son ami Adhémar Raynault est élu maire de Montréal en décembre 1936, il est nommé à la tête du Service d'urbanisme et de recherche de la Commission métropolitaine de Montréal ${ }^{94}$. Poste qu'il occupera pendant environ deux ans. Durant cette période, il s'adonne aussi à la dramaturgie et il participe à la fondation du Mont-Royal Théâtre français Inc. ${ }^{95}$.

89. Archives de Louis Francoeur, Texte d'une causerie prononcée par Louis Francoeur sur les ondes radiophoniques de la station CFCF, 11 août 1936.

90. Archives de Louis Francoeur, Texte d'une causerie prononcée par Louis Francoeur sur les ondes radiophoniques de la station CHLP, 13 août 1936.

91. Voir en particulier la série d'articles "Duplessis intime», dans laquelle Francoeur trace un portrait louangeur du chef de l'Union nationale: Louis Francoeur, "Duplessis intime», La Patrie du Dimanche, série d'articles publiés du 23 août 1936 au 13 septembre 1936.

92. Archives de Louis Francoeur, Lettre d'Antonio Saint-Jean à Louis Francoeur, 15 juin 1936.

93. Archives de Louis Francoeur, Lettre de Louis Francoeur à Joseph L. Boulanger, 10 novembre 1939 .

94. Le mandat de cet organisme est d'organiser la publicité touristique de l'île de Montréal et d'administrer l'aspect de l'esthétique urbaine. Plus concrètement, Francoeur réalise des brochures touristiques, il tente d'inciter les constructeurs à faire appel à des architectes dans leurs projets et il participe à la revalorisation de l'île Sainte-Hélène. Ses archives comprennent différents documents administratifs du Service d'urbanisme et de recherche de la Commission métropolitaine de Montréal.

95. Il s'agit de la section francophone du Montreal Repertory Theatre (M.R.T.). Jusqu'en 1940, Louis Francoeur en sera l'un des deux vice-présidents. Sous la direction de Mario Duliani, l'objectif de cette compagnie de théâtre était de favoriser la production et la présentation de pièces canadiennes-françaises. En 1938, sept pièces sont produites par le MRT français, dont l'une de Francoeur, intitulée Trio à Quatre. "Les activités du M.R.T. Français Inc.», Le M.R.T. Français, 1, 1, 14 décembre 1938, 1. 


\section{JOURNALISTE À RADIO-CANADA, |939-1941}

Louis Francoeur prononçait occasionnellement des causeries politiques à la radio depuis 1935. Par contre, c'est en 1939 qu'il fait ses véritables débuts comme journaliste radio. On lui demande d'abord de décrire et de commenter les activités entourant l'élection pontificale de Pie XII. Puis, lorsque la guerre éclate en Europe, Radio-Canada lui demande de présenter les actualités. Comme il est polyglotte - il maîtrise le français, l'anglais, l'allemand, l'italien et l'espagnol - Francoeur est en mesure de traduire en direct les discours des dirigeants allemands et italiens. Pour s'assurer de l'exclusivité de ses services, Radio-Canada lui confie sa propre émission.

À partir du 25 juin 1940, Francoeur est à la barre de La situation ce soir ${ }^{96}$. D’abord diffusée le samedi, cette émission est ensuite transformée en quotidienne. Il y présente et commente les actualités militaires. Comme l'explique Jacques Francoeur, son père « relatait les orientations diplomatiques, les combats de la journée, le tout agrémenté de commentaires historiques et géographiques ${ }^{97}$ ». De plus, il recevait parfois des invités avec qui il discutait des derniers développements. La situation ce soir est un franc succès; l'émission est écoutée chaque soir par plusieurs milliers d'auditeurs ${ }^{98}$.

Comme le souligne Elzéar Lavoie dans son analyse des fascicules de $\mathrm{La}$ situation ce soir ${ }^{99}$, Francoeur ne se gêne pas pour prendre position. Après la défaite de la France - défaite causée en partie selon lui par les actions communistes de Léon Blum ${ }^{100}$ - il se range sans équivoque dans le camp du général Charles de Gaulle et du mouvement de la France libre. Francoeur se montre très critique envers le maréchal Pétain, le régime de Vichy et les opposants à la libération de la France. En plus de son émission de radio, il écrit chaque semaine dans La Patrie. En juillet 1940, il y explique que les Canadiens doivent se méfier du gouvernement de Vichy, puisque celui-ci est sous l'emprise de l'Allemagne nazie et son but est

96. Les archives de Radio-Canada présentent treize clips radio de La situation ce soir: http: / / archives. radio-canada.ca/emissions/375.

97. Archives de Louis Francoeur, Texte de l'allocution de Jacques Francoeur à la soirée en hommage à Louis Francoeur organisée par le Conseil de Presse, 27 novembre 1989.

98. R. Rumilly, op. cit., 75.

99. Elzéar Lavoie, "La Situation ce soir, essais de Louis Francoeur», dans M. Lemire, dir., op. cit., 914-917.

100. Louis Francoeur, «Un peu de ce qui se passe dans le monde», La Patrie, 23 juin 1940, 42. 
d'établir un régime totalitaire inspiré de l'hitlérisme ${ }^{101}$. Puis, à la radio, il soutient que les Canadiens français ont un rôle à jouer dans la libération de leur ancienne mère patrie ${ }^{102}$. Il invite ses compatriotes à donner des biens et des vêtements aux groupes d'aide à la France libre ${ }^{103}$.

Éventuellement, Francoeur exprime la volonté de s'investir encore plus dans le mouvement de la France libre. Le 7 septembre 1940, il envoie une lettre au général de Gaulle ${ }^{104}$. Dans celle-ci, il lui témoigne son respect et lui offre son appui. Francoeur explique que ses amis et lui-même souhaitent apporter leur contribution à la France libre, mais qu'ils ne savent pas à qui s'adresser. Selon lui, "personne d'autorisé ne leur transmet de consigne $^{105}$ ». Peu de temps après l'envoi de sa lettre, Francoeur est mis en relation avec Élisabeth de Miribel (1915-2005), une jeune Française envoyée de Londres par le général de Gaulle en août 1940 pour recueillir des appuis pour la France libre. Dans l'un de ses rapports, elle présente ainsi le journaliste à son supérieur: «Le plus écouté des Canadiens français. Entièrement pour nous, procède quotidiennement par insinuations de façon fort habile et n'hésite pas à prendre parti à l'occasion d'un document important ou d'une victoire en Afrique ${ }^{106}$. ”

Le 29 mai 1941, en revenant des Laurentides après une réunion de l'équipe du jeu-questionnaire S.V.P. à laquelle participe Francoeur, lui et ses collègues ont un accident d'automobile ${ }^{107}$. Le musicien Léo-Pol Morin, l'abbé Wilfrid Morin et le journaliste Fernand Leclerc décèdent. Francoeur est pour sa part transporté à l'hôpital Saint-Luc à Montréal, où il succombe à ses blessures le $1^{\text {er }}$ juin 1941. Il a été enterré avec la croix de Lorraine à la boutonnière, symbole de la France libre, au cimetière de Côte-des-Neiges ${ }^{108}$.

101. Louis Francoeur, «Un peu de ce qui se passe dans le monde», La Patrie, 14 juillet 1940, 46; Louis Francoeur, «Un peu de ce qui se passe dans le monde», La Patrie, 21 juillet 1940, 44.

102. Louis Francoeur, La situation ce soir, 13 novembre 1940.

103. Louis Francoeur, La situation ce soir, 29 novembre 1940.

104. Archives de Louis Francoeur, Lettre de Louis Francoeur au général Charles de Gaulle, 7 septembre 1940 .

105. Ibid.

106. Lettre d'Élisabeth de Miribel à Geoffroy de Courcel, 11 février 1941, citée dans Frédéric Smith, "La France appelle votre secours». Québec et la France libre, 1940-1945 (Montréal, VLB éditeur, 2012), 74.

107. En plus d'animer La situation ce soir, Louis Francoeur participait le dimanche soir à l'émission S.V.P., diffusée à Radio-Canada, dans laquelle des personnes du public tentaient de déjouer une équipe d'intellectuels en répondant à des questions de culture générale.

108. Archives de Louis Francoeur, Texte de l'allocution de Jacques Francoeur à la soirée en hommage à Louis Francoeur organisée par le Conseil de Presse, 27 novembre 1989. 
Nous souhaitions contribuer à mieux faire connaître l'histoire d'un journaliste marquant de l'histoire du Québec, mais aussi celle d'un influent intellectuel engagé des années 1930. L’itinéraire politico-intellectuel de Louis Francoeur n'est pas banal. Enfant, il habite avec sa mère quelque temps au couvent Notre-Dame-de-Lourdes auprès des religieuses. Le fait d'avoir une socialisation primaire dans un milieu religieux influence sans doute son choix, à 18 ans, de quitter pour la Belgique et de réaliser son noviciat auprès des Bénédictins de la congrégation de Solesmes. Après une formation de quatre ans perturbée par la guerre, il préfère quitter la vie religieuse, ayant de la difficulté à composer avec certains aspects de l'orthodoxie cléricale. Malgré tout, il demeure un intellectuel catholique. Habitant à Paris, il milite dans l'ACJF, il est membre d'un comité d'étude sur le mouvement des idées et il participe au mouvement de la renaissance littéraire catholique, où il exprime sa volonté, en tant que futur écrivain, de participer à la défense et à l'ascension de l'Église catholique.

Lors de son retour au Québec en 1923, Francoeur entreprend une carrière dans le journalisme et il s'engage politiquement du côté des conservateurs. Différentes raisons peuvent expliquer ce choix politique. Tout d'abord, il a peut-être été influencé par sa mère qui entretenait des liens privilégiés avec certains conservateurs influents depuis son passage à la Bourse de Montréal où elle travaillait pour la famille Forget ${ }^{109}$. Par ailleurs, en tant qu'intellectuel catholique, Francoeur devait apprécier la bonne entente qui existait alors entre le Parti conservateur et l'Église catholique. Par contre, l'idéologie traditionaliste ne semble pas l'avoir rejoint. Même dans la littérature, à l'École littéraire de Montréal, il préfère se ranger du côté des exotiques plutôt que de celui des régionalistes. Puis, à la tête du Journal, il propose parfois des idées assez progressistes: faire confiance à la jeunesse, réformer le système d'éducation, etc. Également, il plaide pour une réorganisation du Parti conservateur fédéral dans le but avoué de le moderniser ${ }^{110}$.

Dans les années 1930, Francoeur défend souvent des positions nationalistes. Ses éditoriaux les plus nationalistes sont publiés dans L'Illustration. Dans ceux-ci, il appelle les Canadiens français à reprendre le contrôle de la province. De plus, il pense que l'indépendantisme ne devrait pas être un

109. Les Forget étaient des partisans et de généreux donateurs du Parti conservateur. D’ailleurs, en 1899, Louis-Joseph Forget, le patron d'Ernestine Goulet, a financé la fondation du quotidien Le Journal, qui avait pour mission de défendre les idées et les positions des conservateurs. Francoeur aurait-il choisi de nommer son hebdomadaire de 1929 de la même façon en hommage au Journal des Forget de 1899?

110. Louis Francoeur, «La crise du Parti conservateur», L'Illustration, série d'articles publiés du 27 septembre 1934 au 29 septembre 1934. 
sujet tabou. Sans aller jusqu'à appuyer ouvertement l'idée, il considère qu'il s'agit d'une possibilité à ne pas écarter. En 1935, à la suite d'une mésentente avec Richard B. Bennett sur la défense des droits des francophones au Canada, il quitte le Parti conservateur fédéral pour se joindre à l'éphémère Parti de la reconstruction. Puis, il tente de monter les échelons dans l'Union nationale, mais il est éventuellement écarté par les ténors du parti. Peut-être représentait-il une menace aux yeux de certains?

Après les élections de 1936, Francoeur s'éloigne de la politique active. Il travaille quelque temps à la Ville de Montréal avant d'entreprendre, en 1939, une carrière à la radio. Lors des dernières années de sa vie, le portrait idéologique de Francoeur est clair: il est un gaulliste partisan actif du mouvement de la France libre. Plusieurs conservateurs canadiens-français plus traditionalistes étaient, au début de la guerre, plutôt favorables à l'arrivée du maréchal Pétain et de son programme de «Révolution nationale», dans lequel on retrouvait une apologie des valeurs traditionnelles ${ }^{111}$. Or, cette perception n'est pas du tout partagée par Francoeur.

Louis Francoeur est un conservateur nationaliste et catholique, mais il a montré à quelques reprises qu'il était plus de la frange progressiste que de la mouvance traditionaliste. Francoeur n'a pas eu la carrière politique qu'il désirait, mais il a connu une brillante carrière de journaliste et de communicateur avant son décès prématuré à l'âge de 46 ans. Il a publié des centaines d'éditoriaux, dont certains d'une grande valeur, que nous ne pouvons que souhaiter voir réunis un jour dans une anthologie.

111. Éric Amyot, Le Québec entre Pétain et De Gaulle. Vichy, la France Libre et les Canadiens français (Montréal, Fides, 1999), 365 p. 